Conclusion: The COVID-19 pandemic had an important impact in elderly's mental health. This subject should be addressed by profissionals / caregivers and measures tominimize negative consequences are in order.

\title{
557 - Generalized anxiety disorder in older adults: acceptability of guided self-help by a lay provider and preference among different treatment modalities \\ Anne-Julie Gagné, Philippe Landreville, Patrick Gosselin, Pierre-Hugues Carmichael
}

A cognitive-behavioral guided self-help conducted by lay providers (CBT-GSH-LP) had been shown to be effective in treating anxiety and may help facilitate access to treatment. The first objective of this study was to assess the acceptability the CBT-GSH-LP for the Generalised Anxiety Disorder in adults aged 60 and over. Its acceptability was compared to that of the same treatment conducted by a psychotherapist (CBTGSH-PSY) and to that of a face-to-face cognitive behavioral therapy with a psychotherapist (CBT- PSY). The second objective was to assess the preference of the participants for these treatment modalities. As a secondary objective, variables potentially associated with acceptability or preference were explored, as well as reported reasons for treatment preference. Participants were recruited in community centers and private residences. They had to complete a sociodemographic questionnaire, read descriptions of the three treatments, and complete the Treatment Evaluation Inventory for each one, then to place those treatment in order of preference as well as indicating the reason for their preferred treatment. ANOVAs were performed to identify differences in acceptability scores between the three treatment modalities and proportions were calculated for preferred treatment and reasons associated. CBT-GSH-LP was considered moderately acceptable by participants $(\mathrm{N}=116$; mean age $=$ 70.5 years), although significantly less acceptable than the other two treatment modalities. In addition, the proportion of participants who found CBT-GSH-LP to be at least moderately acceptable was high (59,3\%), although lower than that of the other two treatment modalities (CBT-GSH-PSY: 85,8\%; CBT-PSY: 91,2\%). Consequently, the preferred treatment of participants was CBT-PSY followed by CBT-GSH-PSY, then CBT-GSH-LP. Among participants preferring CBT-GSH-LP, its long-lasting effect, ease of access,

training of the therapist, required patient involvement, and autonomy afforded by treatment were the top reasons. Regarding characteristics, the results show that single and widowed older adults considered CBT-GSH-LP more acceptable than married, divorced, or separated people. Thus, although it is not the preferred treatment modality for older adults, CBT-GSH-LP is acceptable and would benefit from being better known and used for generalized anxiety disorder.

\section{8 - MULTIMODAL EEG-MRI IN THE DIAGNOSIS OF MILD COGNITIVE IMPAIRMENT WITH LEWYBODIES}

Jerry Hai Kok Tan, Julia Schumacher, John-Paul Taylor, Alan Thomas Newcastle University, Newcastle Upon Tyne, GB

\section{Background:}

Differentiating mild cognitive impairment with Lewy bodies (MCl-LB) from mild cognitive impairment due to Alzheimer's disease (MCl-AD) is challenging due to an overlap of symptoms. Quantitative EEG analyses have shown varying levels of diagnostic accuracy, while visual assessment of EEG may be a promising diagnostic method. Additionally, a multimodal EEG-MRI approach may have greater diagnostic utility than individual modalities alone.

\section{Research Objective:}

To evaluate the utility of (1) a structured visual EEG assessment and (2) a machine learning multimodal EEG-MRI approach to differentiate $\mathrm{MCl}-\mathrm{LB}$ from MCl-AD. 